\title{
Correction to: Cardiac Regeneration
}

Masaki leda and Wolfram-Hubertus Zimmermann

\section{Correction to:}

M. leda, W.-H. Zimmermann (eds.), Cardiac Regeneration, Cardiac and Vascular Biology, DOI 10.1007/978-3-319-56106-6

The original version of this volume was revised as it was originally published unnumbered. The revised version now has been numbered; numbering is done following the order of appearance in the book series Cardiac and Vascular Biology.

The updated online version of the book can be found at DOI 10.1007/978-3-319-56106-6 